\title{
Unparticle Physics
}

\section{Citation}

Georgi, Howard. 2007. Unparticle physics. Physical Review Letters 98, no. 22: 221601.

\section{Published Version}

http://dx.doi.org/10.1103/PhysRevLett.98.221601

\section{Permanent link}

http://nrs.harvard.edu/urn-3:HUL.InstRepos:2961663

\section{Terms of Use}

This article was downloaded from Harvard University's DASH repository, and is made available under the terms and conditions applicable to Other Posted Material, as set forth at http:// nrs.harvard.edu/urn-3:HUL.InstRepos:dash.current.terms-of-use\#LAA

\section{Share Your Story}

The Harvard community has made this article openly available.

Please share how this access benefits you. Submit a story.

\section{Accessibility}




\title{
乌s \\ Unparticle Physics
}

\author{
Howard Georgi* \\ Center for the Fundamental Laws of Nature, Jefferson Physical Laboratory, Harvard University, \\ Cambridge, Massachusetts 02138, USA \\ (Received 24 March 2007; revised manuscript received 20 May 2007; published 29 May 2007)
}

\begin{abstract}
I discuss some simple aspects of the low-energy physics of a nontrivial scale invariant sector of an effective field theory - physics that cannot be described in terms of particles. I argue that it is important to take seriously the possibility that the unparticle stuff described by such a theory might actually exist in our world. I suggest a scenario in which some details of the production of unparticle stuff can be calculated. I find that in the appropriate low-energy limit, unparticle stuff with scale dimension $d_{\mathcal{U}}$ looks like a nonintegral number $d_{u}$ of invisible particles. Thus dramatic evidence for a nontrivial scale invariant sector could show up experimentally in missing energy distributions.
\end{abstract}

DOI: 10.1103/PhysRevLett.98.221601

PACS numbers: 11.15.Tk, 14.80.- j

Stuff with nontrivial scale invariance in the infrared (IR) [1] would be very unlike anything we have seen in our world. Our quantum mechanical world seems to be welldescribed in terms of particles. We have a common-sense notion of what a particle is. Classical particles have definite mass and therefore carry energy and momentum in a definite relation $E^{2}=p^{2} c^{2}+m^{2} c^{4}$. In quantum mechanics, this relation becomes the dispersion relation for the corresponding quantum waves with the mass fixing the low-frequency cutoff, $\omega^{2}=c^{2} k^{2}+m^{2} c^{4} / \hbar^{2}$.

Scale invariant stuff cannot have a definite mass unless that mass is zero. A scale transformation multiplies all dimensional quantities by a rescaling factor raised to the mass dimension so a nonzero mass is not scale invariant. A free massless particle is a simple example of scale invariant stuff because the zero mass is unaffected by rescaling. But quantum field theorists have long realized that there are more interesting possibilities - theories in which there are fields that get multiplied by fractional powers of the rescaling parameter (see, for example, [2]). The standard model does not have the property of scale invariance. Many of our particles have definite nonzero masses [3]. But there could be a sector of the theory, as yet unseen, that is exactly scale invariant and very weakly interacting with the rest of the standard model (I will make this precise below). In such an interacting scale invariant sector in four space-time dimensions, there are no particles because there can be no particle states with a definite nonzero mass. Scale invariant stuff, if it exists, is made of unparticles.

But what does this mean? It is clear what scale invariance is in the quantum field theory. Fields can scale with fractional dimensions. Indeed, much beautiful theory is devoted working out the structure of these theories (the huge literature intersects with supersymmetry - for a review see [4], with string theory - for a review see [5], and particularly with the anti-de Sitter space/conformal field theory correspondence (AdS/CFT) — for a review see [6]). But what would scale invariant unparticle stuff actually look like in the laboratory? In spite of all we know about the correlation functions of conformal fields in Euclidean space, it is a little hard to even talk about the physics of something so different from our familiar particle theories. It does not seem a priori very likely that such different stuff should exist and have remained hidden. But this is no reason to assume that it is impossible. We should determine experimentally whether such unparticle stuff actually exists. But how will we know if it we see it? That is one of the questions I address in this Letter.

I discuss a simple scenario in which we can say something simple and unambiguous about what unparticles look like. The tool I use to say something quantitative about unparticle physics is effective field theory (see, for example, [7]). The idea is that while the detailed physics of a theory with a nontrivial scale invariant infrared fixed point is thoroughly nonlinear and complicated, the lowenergy effective field theory, while very strange, is very simple because of the scale invariance. We can use this to understand what the interactions of unparticles with ordinary matter look like in an appropriate limit. Parts of what I have to say are well understood by many experts in scale invariant field theories (see, for example, [5]) [8]. I hope to make it common knowledge among phenomenologists and experimenters. My goal here is not to do serious phenomenology myself, but rather to describe very clearly a physical situation in which phenomenology is possible in spite of the essential strangeness of unparticle theories. And while my motivation is primarily just theoretical curiosity, the scheme I discuss could very well be a component of the physics above the TeV scale that will show up at the LHC. To my mind, this would be a much more striking discovery than the more talked about possibilities of supersymmetry (SUSY) or extra dimensions. SUSY is more new particles. From our four-dimensional point of view until we see black holes or otherwise manipulate gravity, finite extra dimensions are just a metaphor (infinite extra dimensions, however, can have unparticlelike behavior-see [9]). Again 
what we see is just more new particles. We would be overjoyed and fascinated to see these new particles and eventually patterns might emerge that show the beautiful theoretical structures they portend. But I will argue that unparticle stuff with nontrivial scaling would astonish us immediately.

Here is the scheme. The very high-energy theory contains the fields of the standard model and the fields of a theory with a nontrivial IR fixed point, which we will call $\mathcal{B Z}$ (for Banks-Zaks) fields. The two sets interact through the exchange of particles with a large mass scale $M_{u}$. Below the scale $M_{\mathcal{U}}$, there are nonrenormalizable couplings involving both standard model fields and BanksZaks fields suppressed by powers of $M_{\mathcal{U}}$. These have the generic form

$$
\frac{1}{M_{u}^{k}} O_{s m} O_{\mathcal{B Z}}
$$

where $O_{s m}$ is an operator with mass dimension $d_{s m}$ built out of standard model fields and $O_{\mathcal{B} Z}$ is an operator with mass dimension $d_{\mathcal{B} Z}$ built out of $\mathcal{B} Z$ fields. The renormalizable couplings of the $\mathcal{B} Z$ fields then cause dimensional transmutation as scale invariance in the $\mathcal{B} Z$ sector emerges at an energy scale $\Lambda_{\mathcal{U}}$. In the effective theory below the scale $\Lambda_{u}$ the $\mathcal{B Z}$ operators match onto unparticle operators, and the interactions of (1) match onto interactions of the form

$$
\frac{C_{u} \Lambda_{U}^{d_{\mathcal{B}}-d_{u}}}{M_{U}^{k}} O_{s m} O_{U}
$$

where $d_{u}$ is the scaling dimension of the unparticle operator $O_{u}$ [for now we assume for simplicity of presentation that $O_{u}$ is a Lorentz scalar; see (22)]. The constant $C_{u}$ is a coefficient function. We are interested in the operators of the lowest possible dimension, which have the largest effect in the low-energy theory, so we will assume that $O_{\mathcal{U}}$ is one such. The effective field theory interaction (2) is a good starting point in our search for unparticle stuff, for two reasons. Because the $\mathcal{B Z}$ fields decouple from ordinary matter at low energies, the interaction (1) should not effect the IR scale invariance of the unparticle. And (1) seems likely to be allowed experimentally for sufficiently large $M_{\mathcal{U}}$. If $M_{\mathcal{U}}$ is large enough, the unparticle stuff just does not couple strongly enough to ordinary stuff to have been seen. What happens as we lower $M_{u}$ or raise our machine energy and this peculiar stuff can be produced by interactions of ordinary particles?

If the IR fixed point is perturbative, we may be able to calculate the $d_{\mathcal{U}} \mathrm{s}$ and $C_{\mathcal{U}} \mathrm{s}$. But typically the matching from the $\mathcal{B Z}$ physics to the unparticle physics will be a complicated strong interaction problem, like the matching from the physics of high-energy QCD onto the physics of the low-energy hadron states. In that case, we should be able to estimate these constants very roughly by including the appropriate geometrical factors (powers of $4 \pi$ and that sort of thing - we will return to this below), but detailed calculation will be impossible.

Now we can ask what physics this produces in the lowenergy theory below $\Lambda_{u}$. We expect that the virtual effects of fields with nontrivial scaling will produce odd forces. But here I consider what it looks like to actually produce the unparticle stuff. The most important effects will be those that involve only one factor (in the amplitude) of the small parameter in (2),

$$
\frac{C_{u} \Lambda_{u}^{d_{\mathcal{B}}-d_{u}}}{M_{u}^{k}}
$$

from a single insertion of the interaction (2) in some standard model process. The result will be the production of unparticle stuff, which will contribute to missing energy and momentum. To calculate the probability distribution for such a process, we need to know the density of final states for unparticle stuff. In the low-energy theory described above, this is constrained by the scale invariance. Consider the vacuum matrix element

$$
\left\langle 0\left|O_{\mathcal{U}}(x) O_{U^{\dagger}}^{\dagger}(0)\right| 0\right\rangle=\int e^{-i p x}\left|\left\langle 0\left|O_{\mathcal{U}}(0)\right| P\right\rangle\right|^{2} \rho\left(P^{2}\right) \frac{d^{4} P}{(2 \pi)^{4}},
$$

where $|P\rangle$ is the unparticle state with 4-momentum $P^{\mu}$ produced from the vacuum by $O u$. Because of scale invariance, the matrix element (4) scales with dimension $2 d u$, which requires that

$$
\left|\left\langle 0\left|O_{u}(0)\right| P\right\rangle\right|^{2} \rho\left(P^{2}\right)=A_{d u} \theta\left(P^{0}\right) \theta\left(P^{2}\right)\left(P^{2}\right)^{d u^{-2}} .
$$

This is the appropriate phase space for unparticle stuff. (5) should remind you of the phase space for $n$ massless particles [the left-hand side has an extra $(2 \pi)^{4}$ compared to the definition in the particle data book],

$$
(2 \pi)^{4} \delta^{4}\left(P-\sum_{j=1}^{n} p_{j}\right) \prod_{j=1}^{n} \delta\left(p_{j}^{2}\right) \theta\left(p_{j}^{0}\right) \frac{d^{4} p_{j}}{(2 \pi)^{3}}=A_{n} \theta\left(P^{0}\right) \theta\left(P^{2}\right)\left(P^{2}\right)^{n-2},
$$

where

$$
A_{n}=\frac{16 \pi^{5 / 2}}{(2 \pi)^{2 n}} \frac{\Gamma(n+1 / 2)}{\Gamma(n-1) \Gamma(2 n)} .
$$

The zero in $A_{n}$ for $n=1$ together with the pole in $P^{2}$ reproduce the $\delta\left(P^{2}\right)$ in 1-particle phase space if the limit $n \rightarrow 1$ is approached from above

$$
\lim _{\epsilon \rightarrow 0+} \frac{\epsilon \theta(x)}{x^{1-\epsilon}}=\delta(x) .
$$


Thus we can describe the situation concisely as follows:

Unparticle stuff with scale dimension $d_{u}$ looks like a nonintegral number $d_{u}$ of invisible particles.

In fact, we may as well identify the $A$ in (5) with the $A$ in (7), and thus adopt (7) for nonintegral $n$ as the normalization for $A_{d u}$. This is purely conventional because a different definition could be absorbed in the coefficient function $C_{u}$ in (2), but this choice fixes the normalization of the field $O_{\mathcal{U}}$ in a way that incorporates the geometrical factors that go with dimensional analysis, although the combinatoric factors may be wildly wrong.

To illustrate the procedure in a realistic situation consider the decay $t \rightarrow u+\mathcal{U}$ of a $t$ quark into a $u$ quark plus unparticles of scale dimension $d u$ from the coupling (chosen for simplicity rather than interest)

$$
i \frac{\lambda}{\Lambda^{d u}} \bar{u} \gamma_{\mu}\left(1-\gamma_{5}\right) t \partial^{\mu} O_{u}+\text { H.c., }
$$

where the constant $\lambda$

$$
\lambda=\frac{C_{\mathcal{U}} \Lambda_{\mathcal{B}}^{d_{\mathcal{B} Z}}}{M_{\mathcal{U}}^{k}}
$$

(which in this particular case is dimensionless) contains most of the factors from the matching onto the low-energy theory. We can ignore the mass of the $u$ quark, so the final state densities are

$$
\begin{gathered}
d \Phi_{u}\left(p_{u}\right)=2 \pi \theta\left(p_{u}^{0}\right) \delta\left(p_{u}^{2}\right), \\
d \Phi_{u}\left(p_{u}\right)=A_{d_{u}} \theta\left(p_{u}^{0}\right) \theta\left(p_{u}^{2}\right)\left(p_{u}^{2}\right)^{d_{u}-2} .
\end{gathered}
$$

The way the phase space factors compose in my normalization is

$$
d \Phi(P)=\int(2 \pi)^{4} \delta^{4}\left(P-\sum_{j} p_{j}\right) \prod_{j} d \Phi\left(p_{j}\right) \frac{d^{4} p_{j}}{(2 \pi)^{4}}
$$

and the differential decay rate is

$$
d \Gamma=\frac{|\mathcal{M}|^{2}}{2 M} d \Phi(P),
$$

where $\mathcal{M}$ is the invariant matrix element. Suitably averaged over initial spin and summed over final spin this gives

$$
\frac{d \Gamma}{d E_{u}}=\frac{A_{d u} m_{t}^{2} E_{u}^{2}|\lambda|^{2}}{2 \pi^{2} \Lambda_{u}^{2 d u}} \frac{\theta\left(m_{t}-2 E_{u}\right)}{\left(m_{t}^{2}-2 m_{t} E_{u}\right)^{2-d u}} .
$$

We are primarily interested in the shape as a function of $E_{u}$, so we will plot $d \ln \Gamma / d E_{u}$ which has the simple form

$$
\frac{1}{\Gamma} \frac{d \Gamma}{d E_{u}}=4 d_{u}\left(d_{u}^{2}-1\right)\left(1-2 E_{u} / m_{t}\right)^{d u-2} E_{u}^{2} / m_{t}^{2} .
$$

The result is shown in Fig. 1. As $d_{\mathcal{U}} \rightarrow 1$ from above, $d \ln (\Gamma) / d E_{u}$ becomes more peaked at $E_{u}=m_{t} / 2$, matching smoothly unto the kinematics of a 2-particle decay in the limit, as expected from the general principle (9). Obviously, for higher $d_{\mathcal{u}}$ the shape depends sensitively on $d_{u}$, but at least for $d u$ in this range, the calculation appears to make sense. The kind of peculiar distributions of missing energy that we see in Fig. 1 may allow us to discover unparticles experimentally.

The particular operator (10) is flavor changing, and thus may be suppressed by small and unknown flavor factors. But a similar analysis applies to scattering processes due to flavor conserving operators. The most interesting straightforward things to look at, I believe, are the collider phenomenology of

$$
q+\bar{q} \rightarrow G+\mathcal{U} \quad \text { and } \quad q+G \rightarrow q+\mathcal{U}
$$

from the operators

$$
\frac{C_{u} \Lambda_{\mathcal{U}}^{k+1-d_{u}}}{M_{U}^{k}} \bar{q} \gamma_{\mu} q O_{\mathcal{U}}^{\mu}
$$

where $q$ is a left- or right-handed quark, and the LEP constraints on the operators

$$
\frac{C_{u} \Lambda_{u}^{k+1-d_{u}}}{M_{U}^{k}} \bar{e} \gamma_{\mu}\left(1 \pm \gamma_{5}\right) e O_{U}^{\mu}
$$

where the unparticle operator is Hermitian and transverse,

$$
\partial_{\mu} O_{U}^{\mu}=0
$$

The calculation of matrix elements goes the same way except for the tensor structure. For example,

$$
\left\langle 0\left|O_{U^{\mu}}^{\mu}(0)\right| P\right\rangle\left\langle P\left|O_{u}^{\nu}(0)\right| 0\right\rangle \rho\left(P^{2}\right)=A_{d u} \theta\left(P^{0}\right) \theta\left(P^{2}\right)\left(-g^{\mu \nu}+P^{\mu} P^{\nu} / P^{2}\right)\left(P^{2}\right)^{d u^{-2}}
$$

Also amusing is

$$
G+G \rightarrow G+\mathcal{U}
$$

$$
\frac{C_{\mathcal{U}} \Lambda_{\mathcal{U}}^{k-d_{u}}}{M_{U}^{k}} G_{\mu \nu} G^{\mu \nu} O_{\mathcal{U}} \quad \text { and } \quad \frac{C_{\mathcal{U}} \Lambda_{u}^{k-d u}}{M_{U}^{k}} G_{\mu \lambda} G_{\nu}^{\lambda} O_{u}^{\mu \nu}
$$

from the gluon operators 


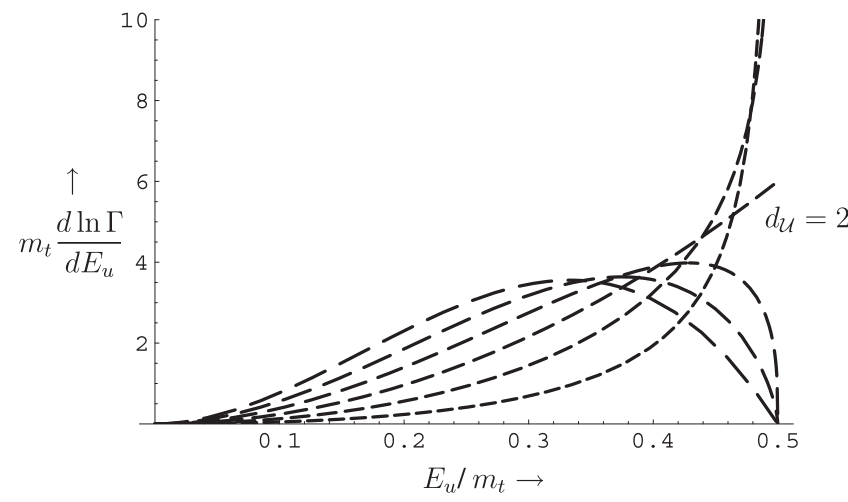

FIG. 1. $\quad d \ln (\Gamma) / d E_{u}$ versus $E_{u}$ in units of $m_{t}$ with $d u=j / 3$ for $j=4$ to 9 . The dashes get longer as $j$ increases.

I have argued in this brief Letter that unparticle stuff with nontrivial scaling dimension might exist in our world, and that up to constants associated with the binding of massless matter into unparticles, we can predict interesting features of unparticle production that serve as experimental tests of this crazy possibility. Let me close with some remarks. (i) Many remarkable things are known about the scale and conformal invariant theories in two dimensions (see, for example, [10]). It is not clear to me what 2D results translate into four dimensions because the phase space in two dimensions is so constrained. But there are certainly consequences for condensed matter physics, where conformal structures do exist (see, for example, [11]). (ii) The connection between operator scaling dimension in a CFT and missing energy distributions was made for ordinary particles with integral scaling dimension in [12]. (iii) The effective field theory picture above assumes that the unparticle fields do not carry the standard model gauge interactions. It would be interesting to try to relax this, but I have no idea whether it is possible. (iv) In (2), (19), (20), and (24) we assumed that the unparticle operator is a bosonic field. Fermionic fields are possible if the standard model fields include fermions and bosons with the same gauge couplings, as in SUSY, or if one can makes sense of unparticle fields with standard model gauge quantum numbers. (v) If unparticles exist, their cosmological consequences should be investigated. It should be possible to use effective field theory to understand how low-energy unparticles behave in the universe today. But additional tools may be required to understand how they got there from the hot big bang. (vi) I had hoped briefly to make sense of unparticles with $d u<1$. However, in the calculation leading to Fig. 1 the differential decay rate into unparticles with $d u<1$ has a nonintegrable singularity as $E_{\mathcal{U}} \rightarrow 0$, suggesting that the vacuum might be unstable. This is in accord with the general theorem in [13] that such fields are not possible in a unitary theory (one of many important contributions by this author to the subject of conformal field theory).

I am grateful to Nima Arkani-Hamed, Tom Banks, Spencer Chang, Ann Nelson, Lisa Randall, and Edward Witten for comments on the manuscript. This research is supported in part by the National Science Foundation under Grant No. PHY-0244821.

*Electronic address: georgi@physics.harvard.edu

[1] T. Banks and A. Zaks, Nucl. Phys. B196, 189 (1982).

[2] K. G. Wilson, Phys. Rev. D 2, 1473 (1970).

[3] It could be that the high energy is scale invariant, but that the scale invariance is broken at or above the electroweak scale. That is not what I am discussing. It leads to ordinary particles.

[4] K. A. Intriligator and N. Seiberg, Nucl. Phys. B, Proc. Suppl. 45, 1 (1996).

[5] O. Aharony, S. S. Gubser, J. M. Maldacena, H. Ooguri, and Y. Oz, Phys. Rep. 323, 183 (2000).

[6] J.M. Maldacena, arXiv:hep-th/0309246.

[7] H. Georgi, Annu. Rev. Nucl. Part. Sci. 43, 209 (1993).

[8] But note that one reason that it is difficult to extract unparticle physics from the beautiful formal works on conformal theory is that these papers often have in mind the scheme described in Ref. [3].

[9] L. Randall and R. Sundrum, Phys. Rev. Lett. 83, 4690 (1999).

[10] P. H. Ginsparg, arXiv:hep-th/9108028.

[11] J. L. Cardy, Ann. Inst. Henri Poincaré, A 4, S371 (2003).

[12] N. Arkani-Hamed, M. Porrati, and L. Randall, J. High Energy Phys. 08 (2001) 017.

[13] G. Mack, Commun. Math. Phys. 55, 1 (1977). 